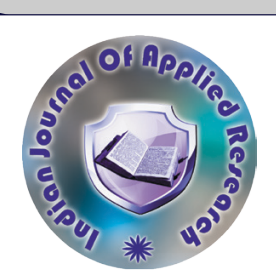

Anesthesiology

\title{
A COMPARISON OF HAND HYGIENE PRACTICES USING "RING THE BELL"; A LOW COST HIGH IMPACT INNOVATIVE TOOL
}

\section{Dr Anand S Kannapur*}

Dr Sudarshan Naik

MD, EDIC, Concept And Design, Drafting \& Manuscript Revision Department Of Anaesthesiology And Critical Care, Command Hospital Air Force Bangalore Agram Post, Bangalore $560007 *$ Corresponding Author

MBBS, Conduct Of Study \& Statistical Analysis Department Of Anaesthesiology And Critical Care, Command Hospital Air Force Bangalore Agram Post, Bangalore 560007

ABSTRACT RATIONALE: Hand hygiene is one of the most neglected practices amongst health care personnel. We devised a positive habit reinforcement tool called "ring the bell" and hypothesized that it will not lead to better hand hygiene compliance.

OBJECTIVES: Hand hygiene events (hand washing and hand rub) of two Intensive Care Units (ICUs) of a large tertiary care hospital were compared.

METHODS: For purpose of the study the control limb was designated ICU A and the test limb ICU B. All medical personnel in ICU B were instructed to "ring the bell" hung next to the wash basin prior to washing hands.

MEASUREMENTS: The study was performed for one hour a day over a period of 90 days and numbers of hand hygiene events were recorded by obscure observers.

MAIN RESULTS: Total hand hygiene events of ICU A were 909 out of $1584(0.58 \%)$ medical personnel attending and in ICU B were 3870 out of $2706(1.43 \%)$ medical personnel attending, a significant increase of more than $125 \%$ ( 2.5 times). The two sided $Z$ test was applied and $Z$ value was calculated to be 12.34 ( $\mathrm{SE}=0.068$ ). Since $\mathrm{p}$ value $(0.0001)$ was less than 0.05 , we rejected the null hypothesis at $5 \%$ level of significance. CONCLUSION(S): "Ring the bell" campaign used for positive habit reinforcement effectively increased hand hygiene compliance and such a low cost and high impact innovative tool is strongly recommended to improve safety and quality of medical care in order to prevent nosocomial infections.

KEYWORDS : Intensive Care Unit; Hand Hygiene; Cross Infections

\section{INTRODUCTION:}

Health Care associated infections (HCAI) occur in approximately one out of ten hospitalized patients. According to the World Health Organization, at any time about 1.4 million people worldwide suffer from HCAI. In developed countries 5-10\% of patients and in developing countries about $15 \%$ patients acquire one or more infections during hospital.(1)

HCAI affects $5-15 \%$ of hospitalized patients in developed countries. The impact on ICU patients is higher with $9-37 \%$ of patients being affected. Rates of hand hygiene compliance have been reported to be either insufficient or very low in both developed and developing countries. Adherence to recommended hand hygiene procedures by health care personnel have been reported variably, with rates ranging from $5 \%$ to $89 \%$ and an average rate of $38.7 \%$.(1)

The burden of HCAI can be directly linked to increased length of hospital stay, morbidity and mortality. It has also resulted in disproportionate increase in cost of medical care, the effects of which are seen both in developed and developing nations. (2)

We started a campaign called "Ring the Bell", in which health care professionals were asked to ring a bell hung next to the wash basin just prior to hand washing. This would produce the sound of a hand bell in the ICU and make other staff working in the ICU aware that someone is washing their hand. The campaign was received with lots of enthusiasm and became a fun activity in the ICU where it was started.

Since it sparked a lot of interest, we decided to study if this intervention would be of help in the real world of hospital infections. Keeping in mind the impact of poor hand hygiene practices on health care; we devised a simple study to see whether this simple, low cost positive habit reinforcement strategy and intervention can affect health care delivery.

We studied hand hygiene practices two Intensive care units in our large tertiary care hospital which had a similar bed capacity, bed occupancy and work load. We introduced ringing the bell before hand washing in one of the ICUs and compared it with the other ICU which acted a control limb.
Studies with such interventions have not been conducted elsewhere. Since this is an issue that is encountered on a daily basis and the subject needed to be effectively addressed, we decided to perform this study.

\section{MATERIALSAND METHODS}

The study was conducted at a large tertiary hospital of over 1000 beds. The hospital has two Intensive Care Units of 13 beds each. The ICUs were designated for the study purpose as ICU A and ICU B. The study was conducted between 01 Jan 2019 and 31 March 2019.

The staffing pattern of both ICUs was similar. ICU A dealt with mainly cardiac patients, both surgical and medical in nature. ICU B was a general ICU which dealt with general medical, surgical and neurosurgical patients.

ICUs A and B had a similar morning routine which included multispecialty ward rounds. Both ICUs were matched with regards to bed occupancy.

The study was performed as an observational case control study. For purpose of the study ICU A was designated as the control limb and ICU $\mathrm{B}$ was designated as the test limb. While the control limb was left as it was, a hand bell was hung next to the wash basin in ICU B with instructions to ring the bell before hand washing (Figure 1). The hand bell was placed a week prior to the start of the study. This was to ensure that all health care personnel working in ICU B got familiar with ringing the bell prior to hand washing and were adhering to the "ring the bell" campaign.

Ring the bel

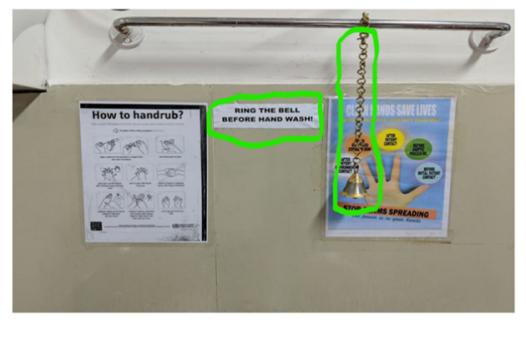

Figure- 1 
Volume-10 | Issue-1 | January - 2020 | PRINT ISSN No. 2249 - 555X | DOI : 10.36106/ijar

After a week of running in of the campaign, when it was thought that the new practice was sufficiently ingrained in the working staff, the study was began. The staff working in either ICUs and visiting teams of doctors was unaware of the study being conducted.

Independent observers (one separate observer for each ICU) from the infection control committee of the hospital who are usually present in the ICUs during the morning rounds were nominated to observe the hand hygiene practices of each ICU.

The independent observer was tasked to note down the number of foot falls in each ICU, hand washing events, hand rubbing events and in addition the observer in ICU B was tasked to note down the number of times the bell was rung prior to washing of hands in. Either hand washing or application of hand rub solution was considered as a hand hygiene event. Hand hygiene index was defined as the total number of hand hygiene events divided by the total number of footfalls in an ICU. The independent observers were instructed to study their respective ICUs for a time period of one hour during the peak time of ICU activity, between $0800 \mathrm{H}$ and $0900 \mathrm{H}$ on each day for a period of 90 days.

\section{STATISTICALANALYSIS}

Statistical software SSPS 13 was used for statistical analysis. The null hypothesis was that there would be no difference in the number of hand hygiene event incidence of both ICUs. The two sided $\mathrm{Z}$ test was applied and the $Z$ value was calculated. Ap value of less than 0.05 was considered significant.

\section{RESULTS}

The health care personnel attending ICUs were categorized as Doctors, Nurses, Trainee Nurses, Medical Assistants and other personnel. Details of the foot falls of health care personnel in each ICU are tabulated (Table 1).

Details Of Footfalls And Hand Hygiene Events Over A 90 Day Period

\begin{tabular}{|l|c|c|}
\hline & ICU A & ICU B \\
\hline Doctors & 369 & 825 \\
\hline Nurses & 603 & 792 \\
\hline Trainee Nurses & 279 & 459 \\
\hline Medical Assistants & 234 & 369 \\
\hline Other personnel & 99 & 261 \\
\hline Total footfall & 1584 & 2706 \\
\hline Hand wash & 396 & - \\
\hline Hand rub & 513 & 810 \\
\hline Hand wash after ringing bell & NA & 2250 \\
\hline Hand wash without ringing bell & NA & 810 \\
\hline Total hand hygiene events & 909 & 3870 \\
\hline $\begin{array}{l}\text { Hand hygiene index (total hand } \\
\text { hygiene events/total footfalls) }\end{array}$ & 0.58 & 1.43 \\
\hline
\end{tabular}

Table-1

The number of foot falls in ICU A was $\mathbf{1 5 8 4}$ health care personnel in the study period. Number of hand washing events in ICU A over the 90 day period was 909 . The hand hygiene index for ICU A was $\mathbf{0 . 5 8 \%}$.

The number of foot falls in ICU B was $\mathbf{2 7 0 6}$ health care personnel. Number of hand washing events in ICU B over the 90 day period was 3870. The hand hygiene index for ICU A was $\mathbf{1 . 4 3 \%}$. Comparison of hand wash events in ICU A and B are depicted in Figure 2. Mean and Standard deviation of hand hygiene index of ICU A and B are depicted in Table 2.

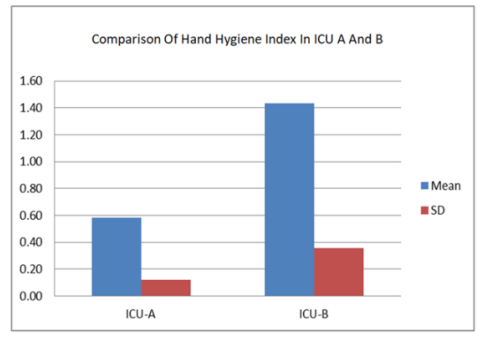

Figure-2
Comparison Of Hand Hygiene Index

\begin{tabular}{|c|c|c|c|c|}
\hline & ICU-A & ICU-B & $\begin{array}{c}\text { Difference } \\
\text { of Means }\end{array}$ & P value \\
\hline Mean & 0.58 & 1.43 & 0.85 & 0.0001 \\
\hline SD & 0.12 & 0.36 & & \\
\hline
\end{tabular}

Table-2

There was a $\mathbf{1 2 5} \%$ increase in hand wash events in ICU B as compared to ICU A which amounted to an increase of $\mathbf{2 . 5}$ times when compared with ICUA.

The two sided $\mathbf{Z}$ test was applied and $\mathbf{Z}$ value was calculated to be $\mathbf{1 2 . 3 4}(\mathbf{S E}=\mathbf{0 . 0 6 8})$. Since $\mathrm{p}$ value $(=\mathbf{0 . 0 0 0 1})$ was less than $\mathbf{0 . 0 5}$ the null hypothesis was rejected at $\mathbf{5 \%}$ level of significance indicating that the "ring the Bell" campaign led to significant increase in hand hygiene events and there by hand hygiene compliance.

\section{DISCUSSION}

Health care associated infections are a dreaded reality. Eighty percent of infectious diseases are transmitted by touch. Such infections are directly related and associated with poor hand hygiene practices. Health care personnel's unclean hands are most often the commonest conduit for transmission of health care associated infection.

Over the past few years many initiatives to facilitate early recognition of sepsis and improving treatment have been made with a high priority accorded to reduce mortality rates and improve outcomes.(3) The $\mathrm{WHO}$ accords high priority to the issue of hand hygiene compliance, and recently came out with its annual call for action to promote hand hygiene. The WHO campaign slogan for the year 2018 is "It's in your hands - prevent sepsis in health care”.(4)

Hand hygiene has been described by the WHO as a generic term referring to any action of hand cleansing.(1) Hand hygiene practices encompass a plethora of terms including antiseptic hand washing, antiseptic hand rubbing, hand care, hand cleansing, hand disinfection, hygienic hand antisepsis, hygienic antisepsis, surgical hand antisepsis, surgical hand scrub, surgical hand rub and the likes.

Generally when the terms hygienic antisepsis are used it refers to eliminating transient flora on hands and not necessarily reducing the skin resident flora. Whereas when the terms surgical hand antisepsis or scrub/rub are used it usually refers to eliminating all transient microflora from the hands and reducing the skin resident flora.

Transient micro-flora are the organisms acquired by health care personnel either from environmental surfaces adjacent to patients or by direct contact with patients and are the organisms usually associated with HCAI.(5)(6) Hands of health care personnel have total bacterial counts that ranged from $3.9 \times 10^{4}$ to $4.6 \times 10^{6} \mathrm{CFU} / \mathrm{cm}^{2}$ as seen by various studies.(7)(8)

In an effort to better hand hygiene practices WHO has actively promoted the "my five moments of hand hygiene" campaign. These include before touching the patient, before clean or aseptic procedures, after body fluid exposure risk, after touching the patient and finally after touching patients surroundings.(1)

There are numerous factors affecting either adherence or nonadherence to good hand hygiene practices. Factors causing poor adherence broadly include observed risk factors, self reported risk factors and additional perceived barriers to appropriate hand hygiene. Factors leading to good adherence include observed factors, predictive factors, self reported factors and factors for preferential recourse to hand rubbing rather than hand washing. (Table 3)

Some Representative Factors Affecting Hand Hygiene Practices

\begin{tabular}{|l|l|l|l|}
\hline \multicolumn{1}{|c|}{ Factors for poor adherence / low compliance } & Factors for good adherence/ improved compliance \\
\hline $\begin{array}{l}\text { a) Observed risk } \\
\text { factors }\end{array}$ & Doctor status & a) Observed factors & $\begin{array}{l}\text { Introduction of widely accessible } \\
\text { alcohol-based hand rub }\end{array}$ \\
\hline & Male sex & $\begin{array}{l}\text { Multifaceted approach to } \\
\text { improve hand hygiene }\end{array}$ \\
\hline & Working in Intensive care & b) Predictive factors & \begin{tabular}{l} 
Non-doctor HCW status \\
\hline
\end{tabular} \\
\hline Working in Anaesthesiology & & $\begin{array}{l}\text { Care of patient under contact } \\
\text { precautions }\end{array}$ \\
\hline & $\begin{array}{l}\text { Understaffing or overcrowding } \\
\text { Intensive care unit }\end{array}$ \\
\hline $\begin{array}{l}\text { b) Self-reported } \\
\text { factors }\end{array}$ & $\begin{array}{l}\text { Hand washing agents cause irritations } \\
\text { and dryness }\end{array}$ & Strong administrative support \\
\hline & $\begin{array}{l}\text { Sinks are inconvenientlylocated or } \\
\text { shortage of sinks }\end{array}$ & $\begin{array}{l}\text { c) Determinants/ } \\
\text { predictors/ self-reported } \\
\text { factors }\end{array}$ & Peer behavior (role model) \\
\hline
\end{tabular}




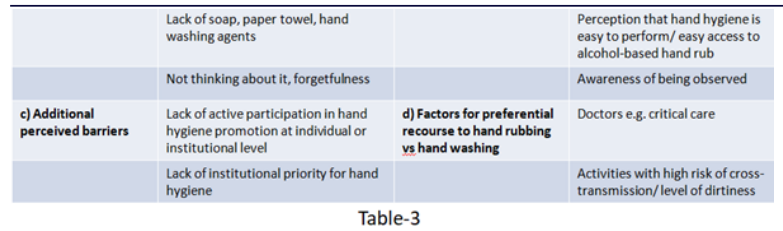

Educational intervention has played a role in hand hygiene compliance improvement but its impact has been variable and inconsistent. In one study, there was an initial improvement in the rate of compliance at one month after the educational intervention [from 89\% (168 of 189 opportunities) to $100 \%$ (212 of 212 opportunities); $P<.001$, but the rate decreased to the baseline rate at three months $[89 \%(85$ of 96 opportunities)].(9) Educational interventions have also been seen to be effective in a paediatric intensive care setting.(10)

Numerous strategies have been looked into to possible improve hand hygiene compliance. These strategies include administrative support, education and training, 'supplies', reminders, performance feedback, and surveillance. One mini systematic review concluded that multi-modal interventions are effective in improving compliance to a particular level after which the effect plateaus out and desired standards are not met. Methodologically good trials of combined strategies and interventions could augment the evidence about interventions to improve hand hygiene compliance among ICU staff.(11)

Multi-modal approaches such as consultation and advocacy meetings, intensifying the provision of alcohol hand rub, training and education, intensifying use of reminders in the workplace, involvement of hospital leaders in hand hygiene improvement, evaluation and feedback and provision and insurance of a continuous supply of related consumables have helped improve hand hygiene compliance.(12) Significantly better hand hygiene practices worldwide have resulted due to Implementation of the WHO multi-modal promotion strategy.(13) Such multi-modal strategies have been applied to infection control practices also.(14)

Hand hygiene protocols also helped in improving hand hygiene compliance as seen by one study which introduced a protocol which possibly shifted the focus from self protection to patient protection.(15)

Behavioral considerations may influence hand hygiene compliance rates. Hand hygiene behavior varies significantly amongst health care personnel within in the same institution or country.(16)(17) Modifying hand hygiene behavior is a complex task and the inability of behavioral modification methods to motivate health care personnel is well known. (18)

We explored the science of behavioural modification in our study to a reasonable extent. Bells sound good and they have been used to great effect at various places. They have been exploited by a very popular food chain which placed a bell near the exit and had a board with the words "ring the bell if you enjoyed yourself". This increased perceived levels of customer satisfaction and translated to increased sales.

This was what sparked the idea for "ring the bell" campaign. Besides hand bells bring in a sense of peace and calm. One usually associates bells with tranquillity and serenity. We thought the pleasant sound of a ringing bell in the ICU would be only a welcome introduction to the existing chaotic environment.

We used a concept called classical conditioning in our study. Conditioning is a process of changing behavior by rewarding or punishing a subject each time an action is performed. Classical conditioning is another term which explains the same concept in greater detail. It is a process in which a previously neutral stimulus evokes a specific response by being repeatedly paired with another stimulus that evokes the response.

In our study the neutral stimulus was the sound of the bell. This sound was repeatedly paired with the process of hand washing. This ended up as a positive behavioral reinforcement loop. Whenever a health care personnel rung the bell and washed hands, it sent a clear message into the environment that a hand hygiene event was being performed.
The hand washer never went unnoticed by others. Being noticed for washing hands by peers, acted as validation for a good act. In addition it acted as constructive feedback for the individual and encouraged him to continue the positively reinforced behavior. This furthermore acted as a trigger or reminder and led more staff either to washing hands or using hand rubs. This also possibly conditioned them to wash hands more frequently.

In our study, though both the ICUs had similar bed strength, bed occupancy and staffing pattern, we observed that the number of footfalls in ICU B exceeded that of ICU A. This was due to the fact that while ICU A had only cardiac patients, ICU B had multi-specialty patients and were visited by treating teams consisting of more number of health care personnel. Hence we used hand hygiene index to compare the two ICUs. We observed that hand hygiene events exponentially increased during the study period by $\mathbf{1 2 5 \%}$, an increase of about $\mathbf{2 . 5}$ times as compared to the control limb. This increase in hand hygiene compliance was sustained over a period of time unlike what has been studied by previous researchers studying other methods to improve hand hygiene compliance.(9)

Our unique study of using this habit reinforcing tool was a very effective low cost, high impact and innovative experiment. This would certainly be well suited for resource limited set ups. Further improvements in the existing tool may enhance its value. For example, a tool that may work even better would be in the form of $\boldsymbol{a}$ bell linked to a digital counter. In this the health care personnel rings an electronic bell and washes her hand. The event gets recorded and displayed digitally for everyone to see. The ICU staff get notified real time of the number of hand washes in the day. Our team is in the process of devising such a hand wash digital counter.

Limitations of the study include it being performed at a single centre. The other limitation of the study was that it was performed for one hour daily for a period of 90 days and this may not be truly representative of the 24 hour hand hygiene events rate and patterns. We also need longer studies to ascertain that the effect of better compliance of hand hygiene practices is sustained. The final limitation was that before starting the study we assumed a similar footfall pattern in both ICUs however on observation we found ICU B had more footfalls than ICU A. We however do not think it would have changed our observations.

\section{CONCLUSION}

Health care associated infections are a common occurrence and good hand hygiene practices can go a long way in decreasing morbidity and mortality associated with them. We developed a simple innovative and effective low cost tool that has a high impact with sustainable improvement in hand hygiene compliance rates. This will be universally helpful in increasing hand wash compliance and would be especially useful in resource limited settings.

\section{REFERENCES}

1. WHO Guidelines on Hand Hygiene in Health Care First Global Patient Safety Challenge Clean Care is Safer Care. 2009.

2. Pittet D, Tarara D, Wenzel RP. Nosocomial Bloodstream Infection in Critically III Patients: Excess Length of Stay, Extra Costs, and Attributable Mortality. JAMA J Am Med Assoc. 1994 May 25;271(20):1598-601.

3. Rhodes A, Evans LE, Alhazzani W, Levy MM, Antonelli M, Ferrer R, et al. SSCG 2016. Vol. 45, Critical Care Medicine. 2017.486-552 p.

4. Masson-Roy S, Saito H, Pittet D. The WHO 2018 hand hygiene campaign: Make a difference-prevent sepsis in health care. American Journal of Respiratory and Critical Care Medicine. 2018 Apr 15;197(8):985-6.

5. Pittet D, Dharan S, Touveneau S, Sauvan V, Perneger T V. Bacterial contamination of the hands of hospital staff during routine patient care. Arch Intern Med. 1999 Apr 26;159(8):821-6.

6. Pessoa-Silva CL, Dharan S, Hugonnet S, Touveneau S, Posfay-Barbe K, Pfister R, et al Dynamics of Bacterial Hand Contamination During Routine Neonatal Care. Infect Control Hosp Epidemiol. 2004 Mar;25(3):192-7.

7. Price PB. The bacteriology of normal skin; a new quantitative test applied to a study of the bacterial flora and the disinfectant action of mechanical cleansing. J Infect Dis. 1938;63(3):301-18.

8. Larson EL, Hughes CAN, Pyrek JD, Sparks SM, Cagatay EU, Bartkus JM. Changes in bacterial flora associated with skin damage on hands of health care personnel. Am J Infect Control. 1998;26(5):513-21.

9. Raskind CH, Worley S, Vinski J, Goldfarb J. Hand hygiene compliance rates after an educational intervention in a neonatal intensive care unit. Infect Control Hosp Epidemiol [Internet]. 2007 Sep [cited 2019 Oct 25];28(9):1096-8. Available from: Epidemiol [Internet]. 2007 Sep [cited 2019 Oct
http://www.ncbi.nlm.nih.gov/pubmed/17932834

10. Nteli C, Galanis P, Koumpagioti D, Poursanidis G, Panagiotopoulou E, Matziou V. Assessing the Effectiveness of an Educational Program on Compliance with Hand Hygiene in a Pediatric Intensive Care Unit. Adv Nurs. 2014;2014:1-4.

11. Alshehari AA, Park S, Rashid H. Strategies to improve hand hygiene compliance among healthcare workers in adult intensive care units: a mini systematic review. Vol. 100, Journal of Hospital Infection. W.B. Saunders Ltd; 2018. p. 152-8.

12. Mahfouz AA, Al-Zaydani IA, Abdelaziz AO, El-Gamal MN, Assiri AM. Changes in hand hygiene compliance after a multimodal intervention among health-care workers hand hygiene compliance after a multimodal intervention among health-care workers
from intensive care units in Southwestern Saudi Arabia. J Epidemiol Glob Health. 2014 


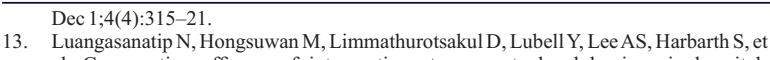
al. Comparative efficacy of interventions to promote hand hygiene in hospital: Systematic review and network meta-analysis. Vol. 351, BMJ (Online). BMJ Publishing Group; 2015.

14. Storr J, Twyman A, Zingg W, Damani N, Kilpatrick C, Reilly J, et al. Core components for effective infection prevention and control programmes: New WHO evidence-based recommendations. Antimicrob Resist Infect Control. 2017 Jan 10;6(1)

15. Fox C, Wavra T, Drake DA, Mulligan D, Bennett YP, Nelson C, et al. Use of a patient Fox C, Wavra T, Drake DA, Mulligan D, Bennett YP, Nelson C, et al. Use of a patient
hand hygiene protocol to reduce hospital-acquired infections and improve nurses' hand washing. Am J Crit Care. 2015;24(3):216-24.

16. Whitby M, McLaws ML. Handwashing in healthcare workers: Accessibility of sink location does not improve compliance. J Hosp Infect. 2004 Dec;58(4):247-53.

17. Gould DJ, Hewitt-Taylor J, Drey NS, Gammon J, Chudleigh J, Weinberg JR. The CleanYourHandsCampaign: critiquing policy and evidence base. Vol. 65, Journal of Hospital Infection. 2007. p. 95-101

18. Pittet D. Improving Compliance With Hand Hygiene in Hospitals. Infect Control Hosp Epidemiol. 2000 Jun;21(6):381-6. 